In der Rubrik "Literatur kompakt" referieren und kommentieren diese Experten für Sie die wichtigsten Originalarbeiten aus der internationalen Fachliteratur.

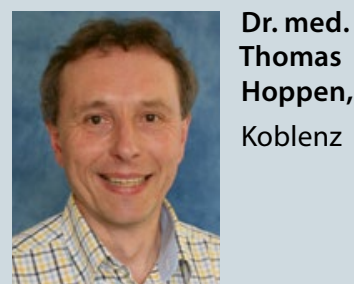

\section{Rate an Neuralrohrdefekten nach wie vor zu hoch}

\section{Vor 20 Jahren ergaben erste Studien belastbare Indizien für den Zusammen- hang zwischen Folsäuremangel und Neuralrohrdefekten. Offensichtlich reicht die Kenntnis dieser Zusammenhänge jedoch nicht aus, um zumindest bei einem Teil von ungeborenen Kindern diese Erkrankung zu verhindern.}

$\mathrm{n}$ einer aktuellen Studie im British Medical Journal wurde die Häufigkeit von Neuralrohrdefekten anhand der Daten der 28 „European Surveillance of Congenital Anomalies"(EUROCAT)-Registers (1991-2011, mit 12,5 Mio. Geburten in 19 europäischen Ländern) untersucht. Dabei ließen sich insgesamt $11.353 \mathrm{Neu}-$

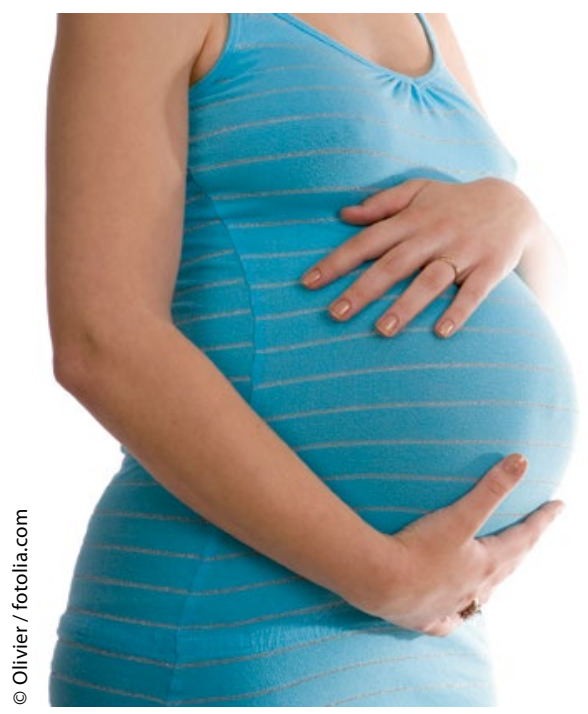

Trotz der Substitutionsempfehlungen von Folsäure bei Schwangeren existiert immer noch eine hohe Rate an Neuralrohrdefekten. ralrohrdefekte feststellen, die ohne Zusammenhang mit chromosomalen Anomalien waren, darunter 4.162 Anenzephalien und 5.776 Kinder mit Spina bifida. Die europäische Gesamtprävalenz betrug damit 9,1/10.000 Schwangerschaften, dabei - bezogen auf die Lebendgeburten jedoch etwas weniger als die Hälfte.

Es ergaben sich im Verlauf des Untersuchungszeitraumes zwar Schwankungen, aber kein signifikanter Rückgang bei den Fallzahlen. Selbst unter Einbeziehung von störenden Faktoren wurden von 1995-1999 jährliche Steigerungen um $4 \%$ beobachtet, gefolgt von einer Reduktion um $3 \%$ in den Jahren 19992003. Danach blieben die Zahlen relativ unverändert, um letztlich im Jahre 2011 die gleiche Häufigkeit wie im Jahre 1991 $\mathrm{zu}$ erreichen. Und auch wenn man Anenzephalie und Spina bifida einzeln analysierte, änderte sich hier an der Häufigkeit nichts Grundlegendes.

Die Autoren um Professeur Babak Khoshnood schreiben in ihrem Kommentar, dass natürlich ein niedriger Folsäurespiegel nicht der einzig mögliche Grund für diese relativ unveränderte Rate an Neuralrohrdefekten sei. Es müssten auch der Nikotinkonsum der
Mutter, chronische Erkrankungen wie Diabetes und bekannte Medikamente wie Antiepileptika als mögliche Risikofaktoren für Neuralrohrdefekte in Betracht gezogen werden, darüber hinaus Veränderungen in der Bevölkerungsstruktur.

Als Konsequenz schreiben die Autoren: Obwohl seit 20 Jahren bekannt sei, dass eine Supplementation mit Folsäure definitiv die Neuralrohrdefektrate senke und auch Schwangere dementsprechend beraten würden, so sei es in Europa nachweisbar nicht gelungen, hier eine effektive Präventionsstrategie zu implementieren. Unter dem Gesichtspunkt einer Nutzen-Risiko-Abwägung sollte daher über eine Folsäureanreicherung in Grundnahrungsmitteln nachgedacht werden.

Khoshnood B et al. Long term trends in prevalence of neural tube defects in Europe: population based study. BMJ 2015;351:h5949

\section{Kommentar}

Es stellt sich auch die Frage, ob im Rahmen einer allgemeinen Schwangerenvorsorge diese Folsäurepräparate nicht kostenlos rezeptiert beziehungsweise über die Krankenkassen abgegeben werden sollten eine entsprechende Kosten-Nutzen-Analyse würde hier sicher zu einem positiven Resultat führen. Insbesondere die Kombination einer frühzeitigen Information mit niedrigschwelliger Versorgung hat sich im Gesundheitswesen bisher immer als positiv herausgestellt (siehe Vitamin-D- und FluorVersorgung).

Dr. Ulrich Mutschler 